\title{
MULTIVARIATE RATIONAL APPROXIMATION
}

\author{
BY \\ RONALD A. DEVORE ${ }^{1}$ AND XIANG - MING YU ${ }^{2}$
}

\begin{abstract}
We estimate the error in approximating a function $f$ by rational functions of degree $n$ in the norm of $L_{q}(\Omega), \Omega:=[0,1]^{d}$. Among other things, we prove that if $f$ is in the Sobolev space $W_{p}^{k}(\Omega)$ and if $k / d-1 / p+1 / q>0$, then $f$ can be approximated by rational functions of degree $n$ to an order $O\left(n^{-k / d}\right)$.
\end{abstract}

1. Introduction. There are many striking results which show that rational functions can approximate much better than polynomials. For example, if $r_{n}(f)$ denotes the error in approximating $f \in C[-1,1]$ by rational functions of degree $n$ in the uniform norm and $E_{n}(f)$ denotes the corresponding error for polynomial approximation, then Newman [7] has shown that $r_{n}(f)$ decreases exponentially (as $\left.n \rightarrow \infty\right)$ when $f(x)=|x|$, but as we know from the results of Bernstein (see [6]) $E_{n}(f) \geqslant c n^{-1}$, $n=1,2, \ldots$. In a slightly different vein, Popov $[\mathbf{8}, 9]$ and Brudnyi $[\mathbf{1}]$ have given classes of functions where rational approximation is better than approximation by polynomials. In fact, they show that for each $\alpha>0$, the class of functions satisfying $r_{n}(f)=O\left(n^{-\alpha}\right)$ is much larger than the class which satisfies $E_{n}(f)=O\left(n^{-\alpha}\right)$. For example Popov [9] proved that when $\alpha=1$, any $f$ with $f^{\prime} \in L_{p}, p>1$, satisfies $r_{n}(f)=O\left(n^{-1}\right)$. For polynomial approximation, we can only get such error when "roughly speaking" $f^{\prime} \in L_{\infty}$.

We are interested in proving multivariate analogues of the results of Popov and Brudnyi. We accomplish this by extending the elementary univariate ideas of DeVore [3] to the multivariate setting. The approach in [3] is to first approximate $f$ by a piecewise polynomial of some small fixed degree consisting of $n$ pieces and then to use a partition of unity of low degree rational functions to join these polynomial pieces into a rational function approximant.

In carrying this approach over to several variables, we encounter two main difficulties. In the univariate case, the piecewise polynomial is constructed by simply "balancing" estimates of the error of polynomial approximation; that is constructing $n$ disjoint intervals $I_{1}, \ldots, I_{n}$, where the errors are about equal. In several variables, such an approach will not lead to cubes. Therefore we replace this first stage by an adaptive approximation which generates cubes $Q_{1}, \ldots, Q_{n}$ on which the error is roughly balanced.

Received by the editors November 19, 1984.

1980 Mathematics Subject Classification. Primary 41A20, 41A25, 41A63.

Key words and phrases. Multivariate rational approximation, error estimates in $L_{q}$, Sobolev spaces.

${ }^{1}$ This author is supported by NSF grant DMS 8320562.

2 This work was completed while this author was a visiting scholar at the the University of South Carolina. 
The second problem we encounter is that in general we cannot piece together local polynomial approximants on such cubes when in the partition $Q_{1}, \ldots, Q_{n}$, large cubes are allowed to neighbor small cubes. For this reason we must modify our adaptive procedure to insure that this does not happen. These results on adaptive approximation are proved in $\$ 3$.

Our results on rational approximation (Theorem 4.1) show the same improvement over polynomial approximation as in the univariate case. For example, it follows from Theorem 4.1 that if $f$ is in the Sobolev space, $W_{p}^{k}(\Omega)$ with $\Omega:=[0,1]^{d}$ the unit cube in $R^{d}$ and $p>d / k$, then $f$ can be approximated in the uniform norm on $\Omega$ by rational function of degree $n$ with an error $O\left(n^{-k / d}\right)$.

The Sobolev spaces are not satisfactory for describing results about rational approximation. For example, we are also interested in the case where the order of approximation is $O\left(n^{-\alpha}\right)$, where $\alpha>0$ is not an integer. This requires the introduction of smoothness spaces of fractional order. But even more importantly, error estimates for rational approximation lead naturally to smoothness spaces in $L_{p}$ for $p<1$. For example, in the univariate case, the best results on rational approximation state that when $f$ has smoothness of order $\alpha$ in $L_{p}$ with $\alpha>1 / p$, then $r_{n}(f)=O\left(n^{-\alpha}\right)$. For this reason, we shall develop our results about rational approximation for the smoothness spaces $C_{p}^{\alpha}$ which were studied by DeVore and Sharpley [5]. These spaces consist "roughly speaking" of functions $f$ such that a fractional derivative $f^{(\alpha)}$ is in $L_{p}$. The precise definition of $C_{p}^{\alpha}$ involves maximal functions and is given in $\$ 2$ together with the properties of these spaces needed for our purposes. The spaces $C_{p}^{\alpha}$ are a generalization of the Sobolev spaces. For example, $C_{p}^{k}=W_{p}^{k}$ if $1<p \leqslant \infty$ and $k$ is an integer. Also $C_{\infty}^{\alpha}$ is the generalized Lipschitz space Lip $\alpha$. Hence our results contain information about approximations of functions from these more classical smoothness spaces.

2. Polynomial approximation. If $f^{(k-1)}$ is absolutely continuous and $f^{(k)} \in L_{p}(I)$, $I:=[0,1]$, then for each $x \in I$, the Taylor polynomial

$$
T_{x}(y):=f(x)+\cdots+f^{(k-1)}(x) \frac{(y-x)^{k-1}}{(k-1) !}
$$

satisfies

$$
\left|f(y)-T_{x}(y)\right| \leqslant|J|^{k-1 / p}\left\|f^{(k)}\right\|_{p}(J)
$$

for any interval $J$ which contains $x$ and $y$. We are interested in generalizations of this result to functions defined on $\Omega:=I^{d}, d \geqslant 1$, which have smoothness of order $\alpha$ (which may be nonintegral) in $L_{p}$, where $p$ may be less than one.

There are well-known results for polynomial approximation of functions of several variables which are somewhat weaker than (2.1). For example, for any cube $Q \subseteq \Omega$, there is a polynomial $P_{Q}$ of degree $<k$ such that

$$
\left\|f-P_{Q}\right\|_{\infty} \leqslant c|Q|^{k / d-1 / p}|f|_{W_{p}^{k}(Q),} \quad 1 \leqslant p \leqslant \infty .
$$


Here $W_{p}^{k}$ denotes the usual Sobolev space with seminorm

$$
|f| W_{p}^{k}(\Omega):=\left(\int_{\Omega}\left|\mathscr{D}_{k} f\right|^{p}\right)^{1 / p}, \quad \mathscr{D}_{k} f:=\sum_{|\nu|=k}\left|D^{\nu} f\right|,
$$

with derivatives taken in the generalized sense.

Estimates of the type (2.2) are known as the Bramble-Hilbert lemma to workers in finite elements but they go back at least to Sobolev [10] in his study of embeddings. The disadvantage of (2.2) when compared with (2.1) is that $P_{Q}$ depends on $Q$ whereas in (2.1) $T_{x}$ depends on $x$, but is independent of the interval. The reason for this is that a function in $W_{p}^{k}$ does not necessarily have ordinary derivatives $D^{\alpha} f$, $|\alpha|<k$, particularly continuous ones, so that the Taylor expansion may not make sense. However an analogue of (2.1) can be given if we replace Taylor derivatives by Peano derivatives. Any function in $W_{p}^{k}, 1 \leqslant p \leqslant \infty$, has Peano derivatives of order $<k$ almost everywhere (see [5, §5]). More generally, such results hold for the spaces $C_{p}^{\alpha}$ which we now define.

The idea behind the spaces $C_{p}^{\alpha}$ is to define a function $f_{\alpha}$ which takes the place of $\mathscr{D}_{k} f$ when $k$ is not an integer and which is meaningful for functions in $L_{p}$ when $p<1$. These functions are defined by their local polynomial approximation. Namely, if $f \in L_{p}(\Omega), p>0$ and $\alpha>0$, we define

$$
f_{\alpha, p}(x):=\sup _{Q \ni x}|Q|^{-\alpha / d} E_{\alpha}(f, Q)_{p}
$$

where

$$
E_{\alpha}(f, Q)_{p}:=\inf _{P \in \mathbf{P}_{\alpha}}\left(\frac{1}{|Q|} \int_{Q}|f-P|^{p}\right)^{1 / p}
$$

is the "averaged" error of approximation to $f$ on $Q$ by polynomials $P \in \mathbf{P}_{\alpha}$, where $\mathbf{P}_{\alpha}$ denotes all polynomials of degree $<\alpha$. When $p=\infty$, the expression on the right side of (2.4) is replaced by $\|f-P\|_{\infty}(Q)$.

When $\alpha=k$ is an integer, $f_{\alpha, p}$ is similar to $\mathscr{D}_{k} f$. In fact, (see [5, p. 33]) we have

$$
\mathscr{D}_{k} f(x) \leqslant f_{k, 1}(x) \leqslant M\left(\mathscr{D}_{k} f\right)(x),
$$

where $M$ is the Hardy-Littlewood maximal operator

$$
M f(x):=\sup _{\Omega \supseteq Q \ni x} \frac{1}{|Q|} \int_{Q}|f| .
$$

For $\alpha, p>0$, we define the space $C_{p}^{\alpha}$ as the set of all $f \in L_{p}(\Omega)$ such that $f_{\alpha, p} \in L_{p}(\Omega)$ and we define its "seminorm" and "norm" by

$$
|f|_{C_{p}^{\alpha}}:=\left\|f_{\alpha, p}\right\|_{p} ; \quad\|f\|_{C_{p}^{\alpha}}:=\|f\|_{p}+|f|_{C_{p}^{\alpha}}
$$

In [5], the definition of $|f|_{C_{p}^{\alpha}}$ uses $f_{\alpha, 1}$ when $p \geqslant 1$, but this is equivalent to the present definition (see [5, Theorem 4.3]). Here and throughout all norms are over $\Omega$ unless otherwise indicated. It can be shown $\left[5, \mathrm{p}\right.$. 37] that $C_{p}^{k}$ is the Sobolev space $W_{p}^{k}$ with equivalent norms provided $1<p \leqslant \infty$ and $k$ is an integer. 
If $f \in C_{p}^{\alpha}$, then $f$ has Peano derivatives $D_{\nu} f(x),|\nu|<\alpha$ for almost every $x$ in $\Omega$. We let

$$
P_{x} f(y):=\sum_{|\nu|<\alpha} D_{\nu} f(x) \frac{(y-x)^{\nu}}{\nu !}
$$

denote the Taylor-Peano polynomial whenever the corresponding derivatives exist. Then $P_{x} f$ is defined for almost all $x$. We can estimate the approximation of $f$ by $P_{x} f$ by using the maximal operator $M_{p}, p>0$, defined for $g \in L_{p}(\Omega)$ by

$$
M_{p}(g):=\left[M\left(|g|^{p}\right)\right]^{1 / p}
$$

with $M$ the Hardy-Littlewood maximal operator (2.6).

Lemma 2.1. Let $0<p \leqslant q$ and $\alpha>0$ satisfy $\alpha / d+1 / q-1 / p>0$. If $f \in C_{p}^{\alpha}$ and $M_{p}\left(f_{\alpha, p}\right)(x)<\infty$, then $P_{x} f$ exists and satisfies

$$
\left\|f-P_{x} f\right\|_{q}(Q) \leqslant c_{0}|Q|^{\alpha / d+1 / q} M_{p}\left(f_{\alpha, p}\right)(x)
$$

for all cubes $Q$ which contain $x$, with a constant $c_{0}$ depending only on $\alpha, p$ and $q$.

For the univariate case, $d=1$, this lemma is Lemma 3.1 of [3]. The proof for $d>1$ is the same. It may be useful to note that the condition $\alpha / d+1 / q-1 / p>0$ which appears in Lemma 2.1 and later is exactly the condition of the Sobolev embedding theorem and guarantees in our case that $f \in C_{p}^{\alpha}$ implies $f \in L_{q}$.

3. Adaptive approximation. Given a function $\phi$ which is in $L_{r}[0,1]$, clearly there is a partition of $[0,1]$ into $n$ intervals $I_{j}$ such that $\int_{I_{j}}|\phi|^{r}=n^{-1} \int_{0}^{1}|\phi|^{r}$. There is no immediate generalization of this to functions in $L_{p}(\Omega), \Omega:=[0,1]^{d}$ when $d>1$. However, if we assume slightly more about $\phi$, namely that $\phi \in L_{\gamma}$ for some $\gamma>r$, then the adaptive technique of Birman and Solomjak [2] will generate a partition of $\Omega$ into cubes $\left(Q_{i}\right)_{1}^{N}$ with $N \leqslant c n$ and $\int_{Q_{i}}|\phi|^{r} \leqslant n^{-1}, i=1, \ldots, n$. We need some additional properties of this partition, most importantly that any neighboring cubes have comparable size. In $\S 4$, we shall apply this adaptive method with $\phi:=M_{p}\left(f_{\alpha, p}\right)$ and thereby generate a good piecewise polynomial approximation to $f$.

Lemma 3.1. Let $0<r<\gamma$. There is a constant $c=c(r, \gamma, d)$ such that for each $\phi \in L_{\gamma}$ with $\|\phi\|_{\gamma}=1$, there is a partition $G$ of $\Omega$ into closed cubes with the following properties:

(iii) $|Q| \leqslant n^{-1}, \quad Q \in G$,

(iv) if $Q \cap Q^{\prime} \neq \varnothing$ with $Q, Q^{\prime} \in G$, then $\left|Q^{\prime}\right| \leqslant 2^{d}|Q|$,

(v) $|G| \leqslant c n$.

Proof. We can assume that $n=m^{d}$ with $m$ a positive integer. Let $I(Q):=\int_{Q}|\phi|^{r}$ and let $\varepsilon:=n^{-1}$. Consider first the partition $P_{0}$ of $\Omega$ into $n$ closed cubes $Q$ of equal size $\left(|Q|=n^{-1}\right)$. We will now select good cubes which will end up in our partition $G$ 
and bad cubes which will need to be further divided. We say a cube $Q \in P_{0}$ is bad if either $I(Q)>\varepsilon$ or $I\left(Q^{\prime}\right)>\varepsilon$ for some $Q^{\prime} \in P_{0}$ which intersects $Q$. We denote the set of all bad cubes in $P_{0}$ by $B_{0}$. All remaining cubes in $P_{0}$ are called good and we let $G_{0}$ denote the set of all good cubes.

We now proceed inductively. Suppose $P_{k}, G_{k}$ and $B_{k}$ have been defined as sets of cubes of measure $2^{-k d} n^{-1}$. We define $P_{k+1}$ as the collection of all cubes which result when the cubes of $B_{k}$ are each subdivided into $2^{d}$ cubes of equal size. We say $Q \in P_{k+1}$ is bad if either $I(Q)>\varepsilon$ or $I\left(Q^{\prime}\right)>\varepsilon$ for some $Q^{\prime} \in P_{k+1}$ which touches $Q$. The collection of bad cubes in $P_{k+1}$ is denoted by $B_{k+1}$ and the collection of the remaining cubes from $P_{k+1}$ is denoted by $G_{k+1}$. We let $G:=\bigcup_{k \geqslant 0} G_{k}$.

Let us next observe that for some $k, B_{k}=\phi$ so that this process terminates. Indeed, from Hölder's inequality,

$$
I(Q)=\int_{Q}|\phi|^{r} \leqslant\left(\int_{Q}|\phi|^{\gamma}\right)^{r / \gamma}|Q|^{1-r / \gamma} .
$$

Since $1-r / \gamma>0, I(Q)<\varepsilon$ provided $|Q|$ is sufficiently small.

We now verify (3.1). Properties (i), (ii) and (iii) are all obvious. To prove property (iv) suppose $Q, Q^{\prime} \in G$ with $Q \in G_{k}$ and $Q^{\prime} \in G_{j}$. If $j \geqslant k$, (iv) is obvious. Suppose then that $j<k$ and let $\tilde{Q}$ be the cube in $P_{j+1}$ which contains $Q$. We will now show that $\tilde{Q} \in G_{j+1}$ which means $Q=\tilde{Q}$ and proves (iv). Now if $R$ is in $P_{j+1}$ and $R$ touches $\tilde{Q}$, then the parent $R_{0} \in P_{j}$ of $R$ must touch $Q^{\prime}$. Hence $I(R) \leqslant$ $I\left(R_{0}\right)<\varepsilon$, as desired.

For the proof of (v), we introduce $B_{j}^{\prime}$ which consists of all $Q \in B_{j}$ such that $I(Q)>\varepsilon$. We now associate to each cube $Q \in G_{j+1}$ a cube $\tilde{Q} \in B_{j}^{\prime}$ in the following way. If $Q_{0} \in B_{j}$ is the parent of $Q$, then for a cube $\tilde{Q} \in P_{j}$ which is either $Q_{0}$ or touches $Q_{0}$ we have $I(\tilde{Q})>\varepsilon$. Clearly $\tilde{Q} \in B_{j}^{\prime}$. Also any $R \in B_{j}^{\prime}$ can be a $\tilde{Q}$ for at most $A_{d}$ cubes $Q \in G_{j+1}$ with $A_{d}$ depending only on $d$; namely $Q$ must be a first generation descendent of $\tilde{Q}$ or of a neighbor of $\tilde{Q}$. Hence

$$
\left|G_{j+1}\right| \leqslant A_{d}\left|B_{j}^{\prime}\right|, \quad j=0,1, \ldots
$$

Now from (3.2) and the definition of $B_{j}^{\prime}$, we have for each $Q \in B_{j}^{\prime}$,

$$
\varepsilon^{\gamma / r} \leqslant I(Q)^{\gamma / r} \leqslant\left(2^{-j d} n^{-1}\right)^{-1+\gamma / r} \int_{Q}|\phi|^{\gamma} .
$$

Summing this over all $Q \in B_{j}^{\prime}$ and using $\varepsilon=n^{-1}$ (and $\|\phi\|_{\gamma}=1$ ) gives

$$
\left|B_{j}^{\prime}\right| \leqslant n\left(2^{-j}\right)^{\tau}, \quad \tau:=d(-1+\gamma / r)>0 .
$$

From (3.3), this gives $\sum_{1}^{\infty}\left|G_{j}\right| \leqslant \sum_{0}^{\infty}\left|B_{j}^{\prime}\right| \leqslant c n$. Since $\left|G_{0}\right|=n$, we have (v). let

$$
r_{n}(f)_{q}:=\inf _{\operatorname{deg}(R) \leqslant n}\|f-R\|_{q},
$$


where the infimum in (4.1) is taken over all rational functions of degree $\leqslant n$, that is all $R=P_{1} / P_{2}$ with $P_{1}, P_{2}$ polynomials of total degree $\leqslant n$. Our main results are:

THEOREM 4.1. If $\alpha, p>0$ and $1 \leqslant q \leqslant \infty$ satisfy $\alpha / d-1 / p+1 / q>0$, then there is a constant $c=c(\alpha, p, q, d)$ such that for all $f \in C_{p}^{\alpha}$, we have

$$
r_{n}(f)_{q} \leqslant c|f|_{C_{p}^{\alpha} n^{-\alpha / d}, \quad n=1,2, \ldots .}
$$

COROllaRY 4.2. If $k$ is a positive integer and $1 \leqslant p, q \leqslant \infty$ satisfy $k / d-1 / p+$ $1 / q>0$, then there is a constant $c=c(k, p, q, d)$ such that for all $f \in W_{p}^{k}$, we have

$$
r_{n}(f)_{q} \leqslant c|f| w_{p}^{k} n^{-k / d}, \quad n=1,2, \ldots .
$$

When $1<p \leqslant \infty$, Corollary 4.2 follows from Theorem 4.1 since $W_{p}^{k}=C_{p}^{k}$ with equivalent seminorms. When $p=1$, it follows from the embedding $W_{1}^{k} \hookrightarrow C_{r}^{k}$ for all $r<1$. The latter can be deduced from Theorem 7.1 of [5] and the embedding $W_{1}^{k} \hookrightarrow B_{r}^{k, r}, r<1$, for Besov spaces.

The remainder of this section will be devoted to the proof of Theorem 4.1. Throughout we will use the notation $c, c_{1}, c_{2}, \ldots$ for constants which depend at most on $p, q, \alpha$ and $d$; however the value of $c$ may differ at each occurrence even within the same expression.

We introduce two auxiliary numbers $r$ and $\gamma$ which satisfy $r<\gamma<p$ and $\alpha / d-1 / r+1 / q>0$. Certainly such numbers exist. Their exact value is not important but they are to be fixed for the remainder of the paper. If $f \in C_{p}^{\alpha}$, we let $F:=M_{p}\left(f_{\alpha, p}\right)$. Since $M_{p}$ maps $L_{p}$ into $L_{r}$ for all $r<p$ (because $M$ maps $L_{1}$ into $L_{s}$ for all $s<1$ ), there is a constant $A$ such that

$$
\|F\|_{\gamma} \leqslant A\left\|f_{\alpha, p}\right\|_{p}=A|f|_{C_{p}^{\alpha}} .
$$

It will be enough to prove our theorem for functions $f$ with $|f|_{C_{p}^{\alpha}}=A^{-1}$. Hence from now on, we assume that

$$
\|F\|_{\gamma} \leqslant 1 \text {. }
$$

We now use the adaptive method of $\S 3$ to generate cubes $\left(Q_{i}\right)_{1}^{N}$ which satisfy Theorem 3.1 for $\phi=F$. Let $x_{i} \in Q_{i}$ be chosen so that

$$
F\left(x_{i}\right) \leqslant\left(\frac{1}{|Q|} \int_{Q_{i}} F^{r}\right)^{1 / r} \leqslant\left(n\left|Q_{i}\right|\right)^{-1 / r}, \quad i=1, \ldots, N .
$$

If $P_{i}:=P_{x_{i}} f$ is the Peano-Taylor polynomial as in $\S 2$, then for any $\tilde{Q}_{i}$ which contains $x_{i}$, we have from Lemma 2.1, with $\tau:=\alpha / d+1 / q$,

$$
\left\|f-P_{i}\right\|_{q}\left(\tilde{Q}_{i}\right) \leqslant c\left|\tilde{Q}_{i}\right|^{\tau} F\left(x_{i}\right) \leqslant c\left(n\left|Q_{i}\right|\right)^{-1 / r}\left|\tilde{Q}_{i}\right|^{\tau} \text {. }
$$

We now use the partition $G$ and the points $\left(x_{i}\right)_{1}^{N}$ to construct a partition of unity consisting of elementary rational functions. Let $a:=[\alpha]+2$ and

$$
\psi_{i}(x):=\left(\frac{\left|x-x_{i}\right|^{2 d}}{\left|Q_{i}\right|^{2}}\right)^{-a}, \quad i=1, \ldots, N
$$


where $|y|$ is the usual Euclidean length of the vector $y$. Then $\psi_{i}$ is a rational function of degree $\leqslant 2 d a$. If we let $\Psi:=\sum_{1}^{N} \psi_{i}$, then $R_{i}:=\psi_{i} / \psi$ has the following properties:

$$
\begin{aligned}
& \sum_{1}^{N} R_{i}(x)=1, \quad x \in \Omega, \\
& \left|R_{i}(x)\right| \leqslant \begin{cases}c, & \text { all } x, \\
c\left(\frac{\left|Q_{i}\right|}{\left|x-x_{i}\right|^{d}}\right)^{2 a}, & x \notin Q_{i} .\end{cases}
\end{aligned}
$$

In fact $\psi_{i} \geqslant c$ on $Q_{i}, i=1, \ldots, N$, and so $\Psi \geqslant c$ on $\Omega$. Hence (ii) follows from (4.7).

Our rational approximation to $f$ is $R:=\sum_{1}^{N} P_{i} R_{i}$. Since $\sum_{1}^{N} P_{i} \psi_{i}$ is a rational function of degree $\leqslant 2 d a \alpha N$ and likewise $\Psi$ is a rational function of degree $\leqslant 2 a d N$, it follows that $\operatorname{deg}(R) \leqslant c n$.

To understand why $R$ is a good approximation to $f$, we note that

$$
\left\|\left(f-P_{i}\right) R_{i}\right\|_{q}\left(Q_{i}\right)<c n^{-\tau}
$$

because of (4.6) and the fact that $\left|Q_{i}\right| \leqslant n^{-1}$. On the other hand, away from $Q_{i}$, the function $\left(f-P_{i}\right) R_{i}$ is dampered by $R_{i}$ since this latter function is small away from $Q_{i}$. Nevertheless adding up these estimates is a bit tricky.

We begin by estimating $\|f-R\|_{q}(Q)$ for an arbitrary cube $Q \in G$. Since $f-R$ $=\sum_{1}^{N}\left(f-P_{i}\right) R_{i}$, we have

$$
\|f-R\|_{q}(Q) \leqslant \sum_{1}^{N}\left\|\left(f-P_{i}\right) R_{i}\right\|_{q}(Q)=\Sigma+\Sigma^{\prime},
$$

where $\Sigma^{\prime}$ is the sum over all $i$ such that $Q_{i} \cap Q \neq \varnothing$ and $\sum$ is the remaining sum.

To estimate $\Sigma^{\prime}$, we let $\tilde{Q}_{i}$ be the smallest cube which contains $Q$ and $x_{i}$. Because of (3.1)(iv), we have $\left|\tilde{Q}_{i}\right| \leqslant c\left|Q_{i}\right|$ and also $\left|Q_{i}\right| \leqslant c|Q|$. Hence from (4.6) and (4.8), we have with $\tau:=\alpha / d+1 / q$

$$
\begin{aligned}
\left\|\left(f-P_{i}\right) R_{i}\right\|_{q}(Q) & \leqslant c\left\|f-P_{i}\right\|_{q}(Q) \leqslant c\left\|f-P_{i}\right\|_{q}\left(\tilde{Q}_{i}\right) \\
& \leqslant c\left(n\left|Q_{i}\right|\right)^{-1 / r}\left|\tilde{Q}_{i}\right|^{\tau} \leqslant C n^{-\tau} .
\end{aligned}
$$

Here the last inequality uses the fact that $\left|Q_{i}\right| \leqslant n^{-1}$. Since the number of terms appearing in $\Sigma^{\prime}$ is less than a constant $c$ (again because of (3.1)(iv)), we have

$$
\Sigma^{\prime} \leqslant C n^{-\tau} \text {. }
$$

With a little more work, we can give a similar estimate for $\sum$. In what follows, we use the notation $\lambda Q$ to denote the cube with the same center as $Q$ and sidelength $\lambda$ times the sidelength of $Q$. Now, for any integer $-\infty<\nu<\infty$, we let $I_{\nu}$ denote those indices $i$ which appear in $\sum$ and for which $\left|Q_{i}\right|=2^{\nu d}|Q|$, and we let $\sigma_{\nu}$ denote that portion of $\sum$ which is the sum over $i \in I_{\nu}$. Furthermore, for any integer $-\infty<\mu<\infty$, 
we let $I_{\nu, \mu}$ denote the set of all $i \in I_{\nu}$ such that $\left(1+2^{\mu-1}\right) Q_{i}$ does not intersect $Q$ but $\left(1+2^{\mu}\right) Q_{i}$ does intersect $Q$ and we let $\sigma_{\nu, \mu}$ be the sum of those terms in $\sigma_{\nu}$ which have index $i \in I_{\nu, \mu}$. To estimate $\sigma_{\nu, \mu}$ we shall use the following

LEMMa 4.3. For any $\mu, \nu$, we have

(i) $I_{\nu, \mu}=\phi$ if $\mu<(-\nu)_{+}-1$,

(ii) $\tilde{Q}_{i}:=4\left(1+2^{\mu}\right) Q_{i}$ contains $Q$ for all $i \in I_{\nu, \mu}$,

(iii) $\quad c_{1} 2^{\mu}\left|Q_{i}\right|^{1 / d} \leqslant|x-y| \leqslant c_{2} 2^{\mu}\left|Q_{i}\right|^{1 / d}, \quad x \in Q, y \in Q_{i}, i \in I_{\nu, \mu}$,

(iv) $\left|I_{\nu, \mu}\right| \leqslant c 2^{\mu d}$.

Proof. To prove (i), we consider two cases. First, if $\nu \geqslant 0$ and $i \in I_{\nu, \mu}$, then from (3.1)(iv), any cube which neighbors $Q_{i}$ has measure $\geqslant 2^{-d}\left|Q_{i}\right|$. Since $Q$ does not neighbor $Q_{i},\left(1+2^{-2}\right) Q_{i}$ cannot touch $Q$. Hence $\mu \geqslant-1$, as desired.

Secondly, we suppose $\nu<0$. In this case any cube which neighbors $Q$ has measure $\geqslant 2^{-d}|Q|=2^{-d} 2^{-\nu d}\left|Q_{i}\right|$. Hence $\left(1+2^{-\nu-2}\right) Q_{i}$ does not touch $Q$ and therefore $\mu \geqslant-\nu-1$. This establishes (i).

For (ii), we note that (i) implies that $|Q|=2^{-\nu d}\left|Q_{i}\right| \leqslant 2^{(\mu+1) d}\left|Q_{i}\right|$. Since $\left(1+2^{\mu}\right) Q_{i}$ touches $Q$, we have $Q \subseteq \tilde{Q}_{i}$. The upper estimate in (iii) follows from (ii) since $\tilde{Q}_{i}$ contains $x$ and $y$ and hence $|x-y| \leqslant \sqrt{d}\left|\tilde{Q}_{i}\right|^{1 / d}$. The lower estimate in (ii) follows from the fact that $\left(1+2^{\mu-1}\right) Q_{i}$ does not touch $Q$.

Finally from (iii) we have that for each $i \in I_{\nu, \mu}, Q_{i}$ is contained in the ball $B(z, \rho)$ with center $z$ the same as the center of $Q$ and with radius $\rho:=c_{2} 2^{\mu}\left|Q_{i}\right|^{1 / d}=$ $c_{2} 2^{\mu} 2^{\nu}|Q|^{1 / d}$. Since the $Q_{i}$ are disjoint and all have measure $\left|Q_{i}\right|=2^{\nu d}|Q|$, we have

$$
\left|I_{\nu, \mu}\right| \leqslant|B(z, \rho)| / 2^{\nu d}|Q| \leqslant c 2^{\mu d} .
$$

We can now estimate $\sigma_{\nu, \mu}$. Let $\tilde{Q}_{i}$ be as in Lemma 4.3. In view of (4.11)(iii), we have (see (4.8))

$$
\left|R_{i}(x)\right| \leqslant c 2^{-2 \mu d a}, \quad x \in Q, i \in I_{\nu, \mu} .
$$

Since $\tilde{Q}_{i}$ contains $Q$ and $x_{i},(4.6)$ gives with $\tau:=\alpha / d+1 / q$

$$
\begin{aligned}
\left\|\left(f-P_{i}\right) R_{i}\right\|_{q}(Q) & \leqslant\left\|R_{i}\right\|_{\infty}(Q)\left\|f-P_{i}\right\|_{q}\left(\tilde{Q}_{i}\right) \\
& \leqslant c 2^{-2 \mu d a}\left(n\left|Q_{i}\right|\right)^{-1 / r}\left|\tilde{Q}_{i}\right|^{\tau} \\
& =c 2^{-2 \mu d a} n^{-1 / r}\left(2^{\mu d}\right)^{\tau}\left|Q_{i}\right|^{\alpha / d-1 / r+1 / q} \\
& =c n^{-1 / r}\left(2^{\nu d}|Q|\right)^{\tau-1 / r}\left(2^{\mu d}\right)^{-2 a+\tau} .
\end{aligned}
$$

If we now sum over all $i \in I_{\nu, \mu}$ and use (4.11)(iv), we get

$$
\sigma_{\nu, \mu} \leqslant c n^{-1 / r}\left(2^{\nu d}|Q|\right)^{\tau-1 / r}\left(2^{\mu d}\right)^{-2 a+\tau+1} .
$$

Now, the exponent of $2^{\mu d}$ on the right side of (4.12) is negative because of the definition of $a(a:=[\alpha]+2)$. Therefore, to evaluate $\sigma_{\nu}$, we need to sum a geometric series for $\mu \geqslant(-\nu)_{+}$. This gives

$$
\sigma_{\nu} \leqslant c n^{-1 / r}\left[2^{\nu d}|Q|\right]^{\tau-1 / r}\left(2^{(-\nu)_{+} d}\right)^{-2 a+\tau+1}, \quad-\infty<\nu<\infty .
$$


If $\nu \geqslant 0$, then $(-\nu)_{+}=0$ and hence (4.13) gives

$$
\sigma_{\nu} \leqslant c n^{-1 / r}\left[2^{\nu d}|Q|\right]^{\tau-1 / r}, \quad \nu \geqslant 0 .
$$

Also, if $M$ is the smallest nonnegative integer such that $2^{M d}|Q|>1 / n$, then from (3.1), we have $I_{\nu}=\varnothing$ and $\sigma_{\nu}=0$ if $\nu \geqslant M$. Hence

$$
\begin{aligned}
\sum_{\nu \geqslant 0} \sigma_{\nu} & \leqslant c n^{-1 / r} \sum_{\nu=0}^{M-1}\left[2^{\nu d}|Q|\right]^{\tau-1 / r} \\
& \leqslant c n^{-1 / r} n^{-\tau+1 / r}=c n^{-\tau} .
\end{aligned}
$$

Similarly, $(-\nu)_{+}=|\nu|$ when $\nu<0$. Since $2 a-\tau-1+\tau-1 / r>0$ and $|Q| \leqslant$ $1 / n$, we have from (4.13),

$$
\sum_{\nu<0} \sigma_{\nu} \leqslant c n^{-1 / r}|Q|^{\tau-1 / r} \leqslant c n^{-\tau}
$$

using this with (4.14) gives $\sum \leqslant c n^{-\tau}$. Hence, from (4.10) and (4.9), we have

$$
\|f-R\|_{q}(Q) \leqslant c n^{-\tau} \text {. }
$$

When $q=\infty$, (4.15) immediately gives Theorem 4.1. When $q<\infty$, we have

$$
\|f-R\|_{q}^{q}=\sum_{Q \in G}\|f-R\|_{q}^{q}(Q) \leqslant c N n^{-\tau q} \leqslant c n^{-\alpha q / d}
$$

because $N \leqslant c n$.

\section{REFERENCES}

1. Yu. Brudnyi, Rational approximation and exotic Lipschitz spaces, in [4, pp. 25-30].

2. M. S. Birman and M. Solomjak, Piecewise-polynomial approximation of functions of the classes $W_{p}^{\alpha}$, Math. USSR-Sb. 2 (1967), 295-317.

3. R. A. DeVore, Maximal functions and their application to rational approximation, CBMS Regional Conf. Ser. Math., no. 3, Amer. Math. Soc., Providence, R. I., 1983, pp. 143-155.

4. R. A. DeVore and K. Scherer (editors), Quantitative approximation, Academic Press, New York, 1980.

5. R. A. DeVore and R. C. Sharpley, Maximal functions measuring smoothness, Mem. Amer. Math. Soc., Vol. 47, No. 293, 1984.

6. G. G. Lorentz, Approximation of functions, Holt, New York, 1966, p. 94.

7. D. J. Newman, Rational approximation to $|x|$, Michigan Math. J. 11 (1964), 11-14.

8. V. Popov, Uniform rational approximation of the class $V_{r}$ and its applications, Acta Math. Acad. Sci. Hungar. 29 (1977), 119-129.

9. , On the connection between rational uniform approximation and polynomial $L_{p}$ approximation of functions, in [4, 267-277].

10. S. L. Sobolev, Applications of functional analysis in mathematical physics, Transl. Math. Monos., vol. 7, Amer. Math. Soc., Providence, R. I., 1963.

Department of Mathematics, University of South Carolina, Columbia, South Carolina 29208

Department of Mathematics, Nanjing Normal University, People’s Republic of China 